\title{
Monitoring environmental cleanliness on two surgical wards
}

Dancer $\mathrm{SJ}^{1 *}$, White $\mathrm{LF}^{1}$, Robertson $\mathrm{C}^{2}$.

Dept. of Microbiology ${ }^{1}$, Southern General Hospital, 1345 Govan Road, Glasgow G51 4TF, Scotland

Department of Statistics \& Modelling Science ${ }^{2}$, University of Strathclyde, Glasgow G1 1XH, Scotland

*Corresponding author: Tel: +44-1355 585000

Department of Microbiology, Hairmyres hospital, Eaglesham road, East Kilbride G75 8RG, Scotland

E-mail: stephanie.dancer@lanarkshire.scot.nhs.uk

Keywords: Hospital cleaning; clinical environment; staphylococci; hospitalacquired infection; surgical ward

Running head: Monitoring environmental cleanliness 


\section{Summary}

Ten hand-touch sites were screened weekly on two surgical wards over two consecutive six-month periods. The results were analysed using hygiene standards, which specify 1) an aerobic colony count (ACC) $>2.5 \mathrm{cfu} / \mathrm{cm}^{2}$, and 2) presence of coagulase-positive staphylococci, as hygiene failures. Sites most often failing the standards were beds and hoist (64\%: 33 of 52 weeks), bedside lockers (62\%: 32 of 52) and overbed tables (44\%: 23 of 52). Methicillin-susceptible/resistant Staphylococcus aureus (MSSA/MRSA) were more often recovered from lockers, overbed tables and beds. Recovery of MSSA/MRSA at any site was significantly associated with an ACC $>2.5 \mathrm{cfu} / \mathrm{cm}^{2}$ from that site $(\mathrm{p}=0.001$; OR: 3.35 (95\% CI $1.79,6.28)$ ). In addition, total ACC's $>2.5 \mathrm{cfu} / \mathrm{cm}^{2}$ each week were significantly associated with weekly bed occupancies $>95 \%$ (p=0.0004; OR: 2.94 (95\% CI 1.44, 6.02)). Higher microbial growth levels from hand-touch sites reflect weekly bed occupancies and indicate a risk for both resistant and susceptible S.aureus. These organisms are more likely to be recovered from near-patient sites on the ward. 


\section{Introduction}

Patients and their visitors believe that the visual appearance of a hospital ward is linked with the risk of infection but there is little evidence for this (Dancer 1999). Whether the hospital environment looks clean or not, it hosts a variety of microorganisms, some of which are multiply resistant to antibiotics (Dancer 1999). Staphylococci, in particular, can survive for long periods in the environment and it is possible that the presence of a staphylococcal reservoir, including methicillinresistant Staphylococcus aureus (MRSA), could represent an infection risk for patients (Dancer 1999; Wagenvoort et al. 2000a; Boyce et al. 1997).

It is known that staphylococci are transmitted between patients, staff and the hospital ward environment (Boyce et al. 1997; Dancer et al. 2006; Hardy et al. 2006; Lidwell 1981). The most probable mode of transmission is via 'hand-touch' sites, since these sites offer a niche to microorganisms deposited from the hands, particularly fingertips (Dancer 2004; Dancer 2007; Bhalla et al. 2004). Examples include near-patient surfaces such as beds, curtains, overbed tables and bedside lockers, and surfaces further away from patients, such as computers, telephone and switches on electrical equipment (Boyce et al. 1997; Dancer 2004; Fitzpatrick et al. 2000; Lemmen et al. 204; Sexton et al. 2006; White et al. 2008).

Thorough and adequate cleaning could potentially eliminate the staphylococcal reservoir and disrupt transmission to patients (Dancer 2007; Fitzpatrick et al. 2000; Hardy et al. 2006; Sexton et al. 2006) but there is little evidence for this at present (Noone \& Griffiths 1971; Rampling et al. 2001). Lack of evidence may be due to 
the fact that there is currently no scientific method for measuring the cleaning process (Dancer 2004). Cleanliness is usually appraised visually but whilst this may address aesthetic concerns, it cannot determine microbial contamination (Griffith et al. 2000). Therefore, standards for surface level cleanliness have been proposed. These are based on internationally recognised standards used by the food industry, and modified to reflect differences between risk management in food preparation and the risk of hospital-acquired infection (HAI) (Dancer 2004; Malik

et al. 2003). An important distinction between the food standards and proposed hospital hygiene standards is that the latter specify surfaces frequently touched by hands. They stipulate identification of potential pathogens from any site, along with quantitative assessment of aerobic colony count (ACC) from hand-touch sites (Dancer 2004).

This project aimed to investigate the microbiological cleanliness of two hospital wards over two consecutive six-month periods, using the proposed standards with methicillin-susceptible S.aureus (MSSA) and MRSA as indicator organisms. We were also keen to examine any relationships between levels of microbial dirt on hand-touch sites, bed occupancy rates and the presence of MSSA/MRSA.

\section{Materials and Methods}

\section{II.1 Study wards}

The two study wards A and B contain twenty-one beds including two side-rooms in a UK teaching hospital. Ward A is situated on the first floor of a 100-year old building with Ward B directly above. Both have the same layout, with two side- 
rooms at the entrance of the ward and the remaining beds positioned in groups of three-five in open cubicles on either side of the main walkway through the ward. Ward A is an acute male surgical ward and ward B is for females, although each occasionally hosts patients of the opposite sex when side-rooms are in short supply. Both wards share medical and ancillary clinical staff (physiotherapy, etc.) and occasionally nursing staff. The wards are cleaned by the same team of domestic staff to the same specification; this comprises a daily mop and vacuum, followed every other weekday by a spray clean, both of which take place in the morning (White et al. 2007). At weekends and out-of-hours, Domestic Services offer spot checks, and there are additional resources for special and outbreak situations. Both wards are subject to the same infection control protocols. Patients are admitted for routine and emergency general surgery from home, Accident and Emergency, Surgical Intensive Care and Surgical High Dependency wards. There were no major outbreaks involving either ward during the year-long study, and no additional infection control initiatives other than routine education to promote hand hygiene.

\section{II.2 Environmental screening}

An environmental audit was performed weekly on ten 'hand-touch' sites; three from bedside areas (patient lockers, overbed tables and bed), three from items of clinical equipment (patient hoist, infusion pump and blood pressure (BP) stand), three from sites at the nurses' work station (computer keyboard, desk and patient notes) and the tenth site was a side-room door handle. For the purposes of statistical analysis, patient lockers, overbed tables and beds were designated as 'near-patient' sites; the remainder as 'far-patient' sites. Screening began on ward B 
for a six-month period and then moved to ward A for a further six months. Sampling took place after the ward had received its routine daily clean, which encompassed floors, bathrooms, kitchen and general ward surfaces. Clinical equipment, lockers and overbed tables are routinely cleaned by nursing staff to a set protocol.

Samples were taken from hand-touch sites on the same ward hoist, BP stand and computer keyboard but from different items representing the remaining sites at varied times and on various days, once weekly throughout each consecutive sixmonthly period. One biomedical scientist organised the screening programme and trained and supervised others to a set protocol.

\section{II.3 Microbiology}

Dipslides were chosen for environmental sampling, having been previously validated in other studies (Griffith et al. 2000; White et al. 2008; Obee et al. 2007). One dipslide were used to sample each site, one side coated with nutrient agar and the other with Baird Parker agar (Biotrace ${ }^{\circledR}$, Bridgend, UK). The slides were pressed gently against the site to be sampled, so that the whole surface of the agar made contact with the site, and this pressure was maintained for a maximum of

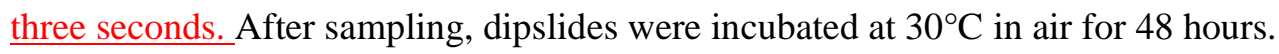
Microbial growth on nutrient agar was quantified as $<2.5 \mathrm{cfu} / \mathrm{cm}^{2}=$ scanty growth; $2.5-12 \mathrm{cfu} / \mathrm{cm}^{2}=$ light growth; $12-40 \mathrm{cfu} / \mathrm{cm}^{2}=$ moderate growth; and 40-

$100 \mathrm{cfu} / \mathrm{cm}^{2}=$ heavy growth, according to manufacturer's recommendations. Black colonies on Baird Parker were identified to genus level by colonial morphology and Gram film. Gram-positive cocci were differentiated by catalase test and 
staphylococci designated S. aureus or coagulase-negative staphylococci (CNS) by Staph-plus (Pastorex ${ }^{\circledR}$, Stockport, UK). Coagulase-positive isolates were subcultured onto MRSA chromogenic agar (Oxoid, UK) and incubated at $37^{\circ} \mathrm{C}$ in aerobic conditions overnight. Antimicrobial susceptibility testing was performed on all staphylococci using the Vitek system standardised in accordance with the Clinical Laboratory Standards Institute (CLSI) guidelines,

\section{II.4 Hygiene status analysis}

The standard set for finding a potential pathogen is $<1 \mathrm{cfu} / \mathrm{cm}^{2}$ (Dancer 2004). For this study, we chose coagulase-positive staphylococci as indicator organisms (Wagenvoort 2000b). A second standard states that the total aerobic colony count (ACC) from a hand-touch site should not exceed 2.5-5 cfu/ $/ \mathrm{cm}^{2}$ (Malik et al. 2003; Dancer 2004). Exceeding these levels suggests insufficient cleaning, masks the presence of a pathogen or implies an increased chance of finding a pathogen with similar epidemiological properties, e.g. CNS and S. aureus (Dancer 2004). A hygiene failure was therefore defined as a site with an ACC greater than $2.5 \mathrm{cfu} / \mathrm{cm}^{2}$ or any hand-touch site demonstrating the presence of MSSA and/or MRSA.

\section{II.5 Statistical analysis}

All variables (higher microbial growth, presence of MSSA and/or MRSA and total hygiene failures) are binary (Yes/No) and were analysed using logistic regression to investigate the relationship between the various types of hygiene failures and ward, sampling day of the week, season of the year, bed occupancy and site. $95 \%$ Confidence intervals for the odds ratio of a hygiene failure are calculated. Exact 
binomial confidence intervals for the percentage of sites with a hygiene fail are calculated. Fisher's Test was used for tests of association.

\section{Results}

Deleted:

II

There was little difference in the overall microbial growth results between the two wards from all ten sites (Table 1). There were more MSSA isolated from Ward B than Ward A (22 isolates vs 8 isolates in six months) but not as many MRSA (seven isolates vs nine isolates). There were no outbreaks of MRSA among patients on either ward during the screening programme, although we did identify a cluster of four patients with MRSA in the second month of screening on Ward A. These patient isolates were sent to the Scottish MRSA Reference laboratory for molecular typing (Macfarlane et al. 1999). This subsequently showed that two strains were indistinguishable by PFGE but different to each of the other two.

Sites most often failing the hygiene standards were patients' beds and the ward hoist (33 of 52 potential failures), lockers (32 of 52) and overbed tables (23 of 52). There were also failures from the nurses' desk and computer keyboard (13 of 52), BP stand (11 of 52), infusion pump and side-room door handle (10 of 52) and patient notes (9 of 52). MSSA was recovered from all sites and MRSA from all except the door handle, infusion pump and patient notes. Patients' lockers, beds and overbed tables were most likely to harbour coagulase-positive staphylococci.

Higher microbial growth $\left(\mathrm{ACC}>2.5 \mathrm{cfu} / \mathrm{cm}^{2}\right)$ at a site was significantly associated with the site $(\mathrm{p}<0.0001)$ and with bed occupancy $(\mathrm{p}=0.0004)$. There was no 
evidence of any difference between the two wards $(\mathrm{p}=0.27)$, nor with the sampling day $(\mathrm{p}=0.31)$ or winter season $(\mathrm{p}=0.53)$. In fact, winter season was confounded with bed occupancy, which is higher in the winter. There is a monotonic association between growth and bed occupancy levels, where the average number of sites per week with a growth failure is 2.4 if occupancy is $<=80 \%$ and 4.3 if occupancy is $95 \%+$ (Table 2). The odds ratio of a growth failure is 2.94 (95\% CI $1.44,6.02)$ times greater if occupancy is $95 \%+, 2.19(1.12,4.27)$ occupancy 90 $95 \%, 1.48(0.75,2.95)$ occupancy $85-90 \%, 0.93(0.44,1.98)$ occupancy $89-85 \%$, compared to $<=80 \%$.

There were more microbial growth failures from beds, lockers and hoist compared to door handle, keyboard, infusion pump, nurses' desk, patient notes and BP stand, with overbed table in between. The three near-patient sites (bed, locker and overbed table) demonstrated significantly more microbial growth than the seven other sites, OR $=4.4(95 \%$ CI 2.9, 6.7), $\mathrm{p}<0.0001$. However, there was still evidence of residual variation between the sites, $\mathrm{p}<0.0001$. The ward hoist, usually stored at the entrance of the ward, demonstrated higher microbial growth levels than the overbed table, which was always near the patient.

MSSA and MRSA were combined in the statistical analysis, as the numbers were small. Among the 352 sites with scanty microbial growth levels $\left(<2.5 \mathrm{cfu} / \mathrm{cm}^{2}\right)$, 5.3\% had additional MSSA/MRSA (95\% CI 3.3\%, 8.3\%), while among the remaining 168 sites demonstrating higher levels of microbial growth, $16.1 \%$ had MSSA/MRSA (95\% CI 10.1\%, 22.5\%). The presence of MSSA/MRSA at a site was significantly associated with light to moderate growth at the same site 
$(\mathrm{p}=0.0001)$. If a site exceeded the microbial growth level standard then the OR of also having MSSA/MRSA was 3.35 (95\% CI 1.79, 6.28).

There was marginal evidence of more MSSA/MRSA found from Ward B compared to Ward A ( $\mathrm{p}=0.06)$ with an OR 1.79 (95\% CI 0.95, 3.34). There was no evidence that MSSA/MRSA was associated with site, bed occupancy, day of the week or season. Adjusting for microbial growth levels and ward, the comparison of near-patient with far-patient sites had an odds ratio of 1.7 (95\% CI 0.9, 3.3), $\mathrm{p}=0.11$. The odds of demonstrating MSSA/MRSA from near-patient sites were $70 \%$ greater than from far-patient sites, but this was not statistically significant. Out of the 156 near-patient sites sampled, 13 were positive for MSSA (8.3\%, 95\% CI $4.5 \%, 13.8 \%)$ and 9 for MRSA $(5.8 \%, 95 \%$ CI $2.7 \%, 10.7 \%)$. Out of the 364 far-patient sites tested 17 were positive for MSSA $(4.7 \%, 95 \%$ CI $2.7 \%, 7.4 \%)$ and 7 for MRSA $(1.9 \% 95 \%$ CI $0.8 \%, 3.9 \%)$. There was no evidence of an association between MRSA or MSSA and near or far-patient site $(\mathrm{p}=0.54)$.

\section{Discussion}

This environmental audit demonstrated the presence of both MSSA and MRSA at hand-touch sites on two acute surgical wards along with varying levels of microbial contamination. The most important finding from the study is the fact that the most heavily contaminated sites were situated right beside patients in their beds. Potential pathogens could be transmitted to patients from hands that have just touched one or more of these contaminated sites (Dancer 2007). In addition, confirmation of the finding that heavier microbial growth from a hand-touch site is 
significantly associated with the presence of coagulase-positive staphylococci suggests that microbial contamination presents an infection risk to patients (White et al. 2007; Griffith et al. 2007). If we assume that more microbes are recovered from a visually dirty surface as opposed to a visually clean one, then the findings from this study support the premise that a patient is more likely to acquire MRSA if resident upon a visually dirty ward. This justifies the current interest in hospital cleaning and suggests that there should be more attention given to basic cleaning of hand-touch sites in clinical wards, particularly those situated beside the patient (Dancer 2007).

Domestic staff are not necessarily responsible for cleaning all the hand-touch sites included in this study. The sites posing the greatest risk, bedside lockers, overbed tables and beds, are routinely cleaned by nurses on the study wards. Trained nurses are usually responsible for cleaning clinical equipment between patient use. In this hospital, domestic staff would only have been responsible for cleaning the sideroom door handle and the top of the nurses' desk.

It has already been suggested that there is an association between high bed occupancy rates and hospital-acquired MRSA (Borg 2003; Cunningham et al. 2006; Kibbler et al. 1998). This study found an association between weekly bed occupancy rates and higher levels of microbial contamination from hand-touch sites. This suggests that busy wards are more likely to be microbiologically dirty, and therefore represent a higher risk of infection to patients. We did not find a significant association between the presence of coagulase-positive staphylococci and bed occupancy rates, but it is possible that another study utilising a more 
frequent and extensive sampling regimen might demonstrate such an association. A recent report from the Department of Health states that the association between MRSA rates and bed occupancy appears to have weakened during 2004-2006 (DOH http://www.dh.gov.uk/publications). Since the analyses were performed using MRSA bacteraemia rates, this finding is not surprising. It would be better to use total MRSA acquisition rates in hospitals when looking at associations with bed occupancy rates. In any case, Trusts now have established protocols for dealing with colonised patients, which would circumvent future invasive sepsis including MRSA bacteraemia.

There has long been dissent over the importance of basic cleaning in a hospital. This study provides more evidence supporting the value of such cleaning in the control of hospital-acquired MSSA and MRSA. We hope that the findings presented here help stimulate future studies examining the effect of targeted cleaning on patient acquisition of MSSA/MRSA in clinical areas.

\section{Acknowledgements}

This study was funded by UNISON, the healthcare workers' union. The authors would like to warmly acknowledge the support from the domestic and microbiological departments at the Southern General Hospital, particularly Mrs Annette McCafferty and Mr John Winning. Additional thanks go to the Scottish MRSA Reference Laboratory in Glasgow, for typing selected MRSA strains. 


\section{References}

1. Bhalla A, Pultz NJ, Gries DM, Ray AJ, Eckstein EC, Aron DC, Donskey CJ. 2004. Acquisition of nosocomial pathogens on hands after contact with environmental surfaces near hospitalised patients. Infect Control Hosp Epidemiol 25: 164-7.

2. Borg MA. 2003. Bed occupancy and overcrowding as determinant factors in the incidence of MRSA infections within general ward settings. J Hosp Infect 54: 316-8.

3. Boyce JM, Potter-Bynoe G, Chenevert C, King T. 1997. Environmental 
contamination due to methicillin-resistant Staphylococcus aureus:

Possible infection control implications. Infect Control Hosp Epidemiol 8: $622-7$

4. Cunningham JB, Kernohan WG, Rush T. 2006. Bed occupancy, turnover intervals and MRSA rates in English hospitals. Br J Nursing 15: 656-60.

5. Dancer SJ. 1999. Mopping up hospital infection. J Hosp Infect 43: 85-100.

6. Dancer SJ. 2004. How do we assess hospital cleaning? A proposal for microbiological standards for surface hygiene in hospitals. J Hosp Infect 56: $10-5$.

7. Dancer SJ, Coyne M, Speekenbrink A, Samavedam S, Kennedy J, Wallace PGM. 2006. Methicillin-resistant Staphylococcus aureus (MRSA) acquisition in an intensive care unit (ICU). Am J Infect Control 34: 10-7.

8. Dancer SJ. 2007. Importance of the environment in meticillin-resistant Staphylococcus aureus acquisition: the case for hospital cleaning. Lancet Infectious Diseases doi.org/10.1016/S1473-3099(07)70241-4.

9. Fitzpatrick F, Murphy OM, Brady A, Prout S, Fenelon LE. 2000. A purpose built MRSA cohort unit. J Hosp Infect 46: 271-9.

10. Griffith CJ, Cooper RA, Gilmore J, Davies C, Lewis M. 2000. An evaluation of hospital cleaning regimes and standards. J Hosp Infect 45: 1928.

11. Griffith CJ, Obee P, Cooper RA, Burton NF, Lewis M. 2007. The effectiveness of existing and modified cleaning regimens in a Welsh hospital. J Hosp Infect 66: 352-59.

12. Hardy KJ, Oppenheim BA, Gossain S, Gao F, Hawkey PM. 2006. A study of the relationship between environmental contamination with methicillin- 
resistant Staphylococcus aureus (MRSA) and patients' acquisition of MRSA. Infect Control Hosp Epidemiol 27:127-32.

13. Kibbler CC, Quick A, O'Neill AM. 1998. The effect of increased bed numbers on MRSA transmission in acute medical wards. J Hosp Infect 39: 213-9.

14. Lemmen SW, Hafner H, Zolldan D, Stanzel S, Lutticken R. 2004.

Distribution of multi-resistant Gram-negative versus Gram-positive bacteria in the hospital inanimate environment. J Hosp Infect 56: 191-7.

15. Lidwell OM. 1981. Some aspects of transfer and acquisition of Staphylococcus aureus in hospitals. In: Macdonald A, Smith G, eds. The Staphylococci. Aberdeen: Aberdeen University Press, 175-200.

16. Macfarlane L, Walker J, Borrow R, Oppenheim BA, Fox AJ. 1999. $\underline{\text { Improved recognition of MRSA case clusters by the application of }}$ $\underline{\text { molecular subtyping using pulsed-field gel electrophoresis. J Hosp Infect }}$ 41: 29-37.

17. Malik RE, Cooper RA, Griffith CJ. 2003. Use of audit tools to evaluate the efficacy of cleaning systems in hospitals. Am J Infect Control 31: 181-7.

18. Noone P, Griffiths RJ. 1971. The effects of sepsis rates of closing and cleaning hospital wards. J Clin Pathol 24: 721-5.

19. Obee P, Griffith CJ, Cooper RA, Bennion NE. 2007. An evaluation of different methods for the recovery of meticillin-resistant Staphylococcus aureus from environmental surfaces. J Hosp Infect 65: 35-41.

20. Rampling A, Wiseman S, Davis L, Hyett AP, Walbridge AN, Payne GC, Cornaby AJ. 2001. Evidence that hospital hygiene is important in the control of methicillin-resistant Staphylococcus aureus. J Hosp Infect 49: 
$109-16$.

21. Sexton T, Clarke P, O’Neill E, Dillane T, Humphreys H. 2006.

Environmental reservoirs of methicillin-resistant Staphylococcus aureus in isolation rooms: correlation with patient isolates and implications for hospital hygiene. J Hosp Infect 62: 187-94.

22. Wagenvoort JHT, Sluijsmans W, Penders RJR. 2000a. Better environmental survival of outbreak versus sporadic MRSA isolates. J Hosp Infect 45: 231-4.

23. Wagenvoort JHT. 2000b. Dutch measures to control MRSA and the expanding European Union. Eurosurveillance 5(3): 26-8.

24. White LF, Dancer SJ, Robertson. 2007. A microbiological evaluation of cleaning methods. Int J Environ Health Res 17: 1-11.

25. White LF, Dancer SJ, Robertson C, McDonald J. 2008. Are hygiene standards useful in assessing infection risk? In press, Am J Infect Control. 
Table Legends

Table 1: Environmental screening results from two acute surgical wards over two consecutive six-month periods

Table 2: Total weekly microbial growth failures $\left(>2.5 \mathrm{cfu} / \mathrm{cm}^{2}\right)$ associated with weekly bed occupancy rates on two surgical wards over two consecutive six-month periods 
Table 1: Environmental screening results from two acute surgical wards over

two consecutive six-month periods

\begin{tabular}{|c|c|c|c|c|c|c|}
\hline$\underline{\text { Site }}$ & Ward & $\begin{array}{l}\frac{\text { Microbial }}{\text { Growth }} \\
\frac{>2.5}{\mathrm{cfu} / \mathrm{cm}^{2}}\end{array}$ & $\underline{\text { MSSA }}$ & MRSA & $\begin{array}{r}\text { Any MSSA } \\
\text { or MRSA }\end{array}$ & $\begin{array}{r}\text { Any } \\
\text { Hygiene Fail }\end{array}$ \\
\hline \multirow[t]{2}{*}{ Bedside Locker } & $\underline{\mathrm{A}}$ & 17 & $\underline{0}$ & 1 & 1 & 17 \\
\hline & B & 15 & $\underline{6}$ & $\underline{1}$ & 7 & 15 \\
\hline \multirow[t]{2}{*}{ Bed Frame } & $\underline{\mathrm{A}}$ & $\underline{20}$ & $\underline{2}$ & $\underline{4}$ & $\underline{6}$ & $\underline{20}$ \\
\hline & $\underline{B}$ & $\underline{13}$ & 1 & $\underline{0}$ & $\underline{1}$ & $\underline{13}$ \\
\hline \multirow[t]{2}{*}{ Overbed Table } & $\underline{\mathrm{A}}$ & $\underline{11}$ & $\underline{2}$ & $\underline{1}$ & $\underline{3}$ & $\underline{11}$ \\
\hline & $\underline{B}$ & $\underline{10}$ & $\underline{2}$ & $\underline{2}$ & $\underline{4}$ & $\underline{12}$ \\
\hline \multirow[t]{2}{*}{$\underline{\text { Hoist }}$} & $\underline{\mathrm{A}}$ & $\underline{13}$ & $\underline{0}$ & $\underline{2}$ & $\underline{2}$ & $\underline{14}$ \\
\hline & B & 18 & $\underline{3}$ & $\underline{0}$ & $\underline{3}$ & 19 \\
\hline \multirow[t]{2}{*}{ Door handle } & $\underline{\mathrm{A}}$ & $\underline{3}$ & $\underline{2}$ & $\underline{0}$ & $\underline{2}$ & $\underline{5}$ \\
\hline & $\underline{B}$ & $\underline{4}$ & 1 & $\underline{0}$ & $\underline{1}$ & $\underline{5}$ \\
\hline \multirow[t]{2}{*}{ Keyboard } & $\underline{\mathrm{A}}$ & $\underline{5}$ & $\underline{0}$ & $\underline{0}$ & $\underline{0}$ & $\underline{5}$ \\
\hline & $\underline{B}$ & $\underline{7}$ & $\underline{2}$ & 1 & $\underline{3}$ & $\underline{8}$ \\
\hline \multirow[t]{2}{*}{ Infusion Pump } & $\underline{\mathrm{A}}$ & $\underline{7}$ & $\underline{0}$ & $\underline{0}$ & $\underline{0}$ & 7 \\
\hline & $\underline{B}$ & 1 & $\underline{2}$ & $\underline{0}$ & $\underline{2}$ & $\underline{3}$ \\
\hline \multirow[t]{2}{*}{ Nurses Station } & $\underline{\mathrm{A}}$ & 2 & $\underline{1}$ & $\underline{1}$ & $\underline{2}$ & $\underline{4}$ \\
\hline & $\underline{B}$ & $\underline{5}$ & $\underline{3}$ & $\underline{1}$ & $\underline{4}$ & $\underline{9}$ \\
\hline \multirow[t]{2}{*}{ Patient Notes } & $\underline{\mathrm{A}}$ & $\underline{3}$ & $\underline{0}$ & $\underline{0}$ & $\underline{0}$ & $\underline{3}$ \\
\hline & $\underline{B}$ & $\underline{5}$ & $\underline{1}$ & $\underline{0}$ & $\underline{1}$ & $\underline{6}$ \\
\hline \multirow[t]{2}{*}{ BP Stand } & $\underline{\mathrm{A}}$ & $\underline{1}$ & $\underline{1}$ & $\underline{0}$ & $\underline{1}$ & $\underline{2}$ \\
\hline & $\underline{B}$ & $\underline{8}$ & 1 & $\underline{2}$ & $\underline{3}$ & $\underline{9}$ \\
\hline
\end{tabular}

$\underline{\text { NB. Some sites with } \mathrm{ACC}>2.5 \mathrm{cfu} / \mathrm{cm}^{2} \text { also demonstrated the presence of }}$ methicillin-susceptible or methicillin-resistant S.aureus (MSSA/MRSA) 
Table 2: Total weekly microbial growth failures $\left(>2.5 \mathrm{cfu} / \mathrm{cm}^{2}\right)$ associated with

$\underline{\text { weekly bed occupancy rates on two surgical wards over two consecutive }}$

$\underline{\text { six-month periods }}$

\begin{tabular}{|c|c|c|c|c|c|c|c|}
\hline \multirow{2}{*}{$\begin{array}{l}\text { Bed } \\
\text { Occupancy }\end{array}$} & \multirow{2}{*}{$\begin{array}{l}\text { Number } \\
\text { Weeks }\end{array}$} & \multicolumn{3}{|c|}{ Total Growth failures } & \multicolumn{3}{|c|}{ Any MRSA or MSSA } \\
\hline & & Mean & $\frac{95 \%}{\mathrm{LCL}}$ & $\begin{array}{l}95 \% \\
\text { UCL }\end{array}$ & Mean & $\begin{array}{l}95 \% \\
\text { LCL }\end{array}$ & $\begin{array}{l}95 \% \\
\mathrm{UCL} \\
\end{array}$ \\
\hline$\leq=80 \%$ & $\underline{9}$ & 2.4 & 1.6 & $\underline{3.5}$ & 1.2 & $\underline{0.6}$ & 2.1 \\
\hline $80-85 \%$ & $\underline{9}$ & 2.3 & 1.5 & 3.3 & 0.7 & $\underline{0.2}$ & 1.4 \\
\hline $85-90 \%$ & 12 & 3.1 & 2.3 & 4.0 & $\underline{0.8}$ & $\underline{0.4}$ & 1.5 \\
\hline $90-95 \%$ & 13 & 3.8 & 2.9 & 4.7 & $\underline{0.7}$ & $\underline{0.3}$ & 1.3 \\
\hline $95 \%+$ & $\underline{9}$ & 4.3 & 3.3 & 5.4 & 1.1 & $\underline{0.5}$ & 1.9 \\
\hline
\end{tabular}

NB. Entries are the mean number of sites per week with microbial growth or MSSA/MRSA failures from 10 hand-touch sites. The higher the bed occupancy rate, the more microbial growth is recovered from hand-touch sites. 